\title{
Evaluación de conocimientos sobre el manejo de ventilación mecánica en médicos residentes
}

\author{
Sergio E. Prieto-Miranda, Rubén A. Calixto-Bello, Carlos A. Jiménez-Bernardino, Laura G. Guerrero-Quintero
}

Introducción. En nuestro medio, los pacientes que requieren ventilación mecánica se encuentran en áreas donde la mayoría de las veces no se dispone de un especialista en cuidados intensivos, quedando a cargo de médicos residentes. El objetivo de este estudio fue validar un instrumento al castellano para conocer el nivel de conocimientos que tienen los residentes sobre ventilación mecánica.

Sujetos y métodos. Se realizó una prueba piloto donde se incluyeron médicos residentes en diferentes años de formación de las áreas de anestesiología, medicina interna y urgencias médico-quirúrgicas, previo consentimiento informado. Se aplicó el cuestionario diseñado por Cox y traducido al castellano para su validación. Éste se basó en cuatro casos clínicos con 19 preguntas.

Resultados. Se aplicaron 81 cuestionarios, de ellos el $46 \%$ correspondieron a médicos de urgencias, el 37\% a médicos de medicina interna y el $17 \%$ a médicos de anestesiología. La mitad de los residentes fueron de primer año. La calificación mínima fue de cuatro aciertos, y la máxima, de 17. El 65\% de los médicos residentes tuvieron calificación no aprobatoria y sólo el $4 \%$ alcanzó un nivel de conocimientos suficiente. No se detectaron dificultades en la aplicación y comprensión de la prueba.

Conclusiones. La complejidad de la prueba fue aceptable y su mayor utilidad fue para médicos residentes en sus últimos años de formación. El instrumento resulta útil para evaluar conocimientos en ventilación mecánica en su versión al casteIlano. El nivel de conocimientos fue insuficiente en la muestra estudiada.

Palabras clave. Médicos residentes. Nivel de conocimientos. Prueba piloto. Ventilación mecánica.

\section{Assessment of knowledge in the management of mechanical ventilation in medical residents}

Introduction. In our environment patients who require mechanical ventilation are in areas where most of the time there is no specialist in intensive care, many patients are the responsibility of medical residents. The objective of this research was to validate an instrument to Spanish to determine the level of knowledge among residents in mechanical ventilation.

Subjects and methods. A pilot test was performed which included medical residents from different years of training in the areas of Anesthesiology, Internal Medicine and Emergency Medicine, prior signed informed consent. We applied the questionnaire designed by Cox and translated from English into Spanish for validation. It was based on four clinical cases with 19 questions.

Results. 81 questionnaires were applied, of whom 46\% were Emergency medicine residents, 37\% of Internal Medicine and $17 \%$ of Anesthesiology. Half of the residents were a first year. The minimum score was 4 and the maximum of 17 . Sixty-five per cent of the residents did not reach a passing score, and only $4 \%$ with an adequate level of knowledge. Not detected difficulties in implementation and understanding of the test.

Conclusions. The complexity of the test was acceptable and was most useful in medical residents in their final years of training. The instrument is useful to assess knowledge on mechanical ventilation in the Spanish version. Level of knowledge was low in our sample studied.

Key words. Level of knowledge. Mechanical ventilation. Pilot test. Resident physicians.

Nuevo Hospital Civil Doctor Juan I. Menchaca (S.E. Prieto-Miranda). Hospital General Regional n.․ 46 'Lázaro Cárdenas'; Instituto Mexicano del Seguro Social (S.E. Prieto-Miranda, R.A. CalixtoBello). Hospital de Especialidades del Centro Médico Nacional de Occidente (C.A. Jiménez-Bernardino) Centro de Investigación Educativa y Formación Docente; Instituto Mexicano del Seguro Social (L.G. Guerrero-Quintero). Guadalajara, Jalisco. México.

Correspondencia:

Dr. Sergio Emilio Prieto Miranda. Hospital General Regional n.․ 46 'Lázaro Cárdenas'. Avda. Lázaro Cárdenas, 2063. Colonia Morelos. CP 44910. Guadalajara, Jalisco, México.

E-mail:

sergio.prieto@imss.gob.mx

Conflicto de intereses: No declarado.

Competing interests: None declared.

(c) 2013 FEM 


\section{Introducción}

En la actualidad, la sociedad reclama médicos que sean lo suficientemente honestos y actúen de acuerdo con los intereses de sus pacientes, para lo cual se ha promovido el desarrollo de competencias en los estudiantes de posgrado. Algunos componentes de estas competencias incluyen habilidades de comunicación, interpersonales y profesionalismo médico, con los cuales los mismos residentes sean capaces de demostrar mejoras en su proceso de educación continua y, de forma paralela, su capacidad de tomar decisiones clínicas [1]. De manera simultánea, en los procesos de certificación hospitalaria se insiste en que los estudiantes de posgrado respeten la autonomía de los pacientes, aprendan a escucharlos, les informen de la evolución de su enfermedad, tomen en cuenta sus preferencias y se involucren en su tratamiento [2]. Para el desarrollo de esto, los residentes necesitan saber cómo tratar adecuadamente a sus pacientes a través de programas de educación efectivos [3].

Se han propuesto diversos mecanismos para mejorar el proceso formativo en los médicos residentes, como son la enseñanza tutorial, el desarrollo de habilidades del pensamiento y el desarrollo de habilidades para la lectura crítica, proponiéndose de manera adicional un sistema de evaluación integral [4]. También se ha puesto énfasis en rediseñar las experiencias curriculares con el fin de lograr mejores resultados, que al desarrollarse la enseñanza tutorial el profesor responsable podría realizar [5]. En nuestro medio, los programas por competencias no establecen claramente en qué momento el profesor puede realizar sus ajustes en el currículo. La estructura básica reconocida de la formación por competencias incluye seis competencias generales: conocimientos médicos, atención médica, habilidades de comunicación e interpersonales, profesionalismo, mejoras en el aprendizaje basado en la práctica médica y sistemas basados en la práctica. Sin embargo, es evidente que la falta de profesionalización de muchos de los docentes no permite una adecuada aplicación de estos programas [6]. La formación de los médicos residentes representa de manera evidente un coste económico, desde su contratación y el pago de su beca hasta el tiempo invertido en su formación, los recursos requeridos para su manutención académica y el otorgamiento de sus prestaciones a las que tienen derecho por ley; además, los costes de atención médica son muy superiores cuando se comparan grupos de pacientes atendidos por residentes con grupos de pacientes que no son atendidos por médicos residentes [7]. Sin embargo, al evaluar el desarrollo de las competencias, este proceso continúa hasta el momento siendo realmente difícil, entendiendo como competencia lo que el residente es capaz de hacer; también debe demostrar capacidad para adaptarse a los cambios, así como encontrar formas que mejoren sus habilidades. Para esto se han propuesto exámenes de opción múltiple, evaluación supervisada, observación directa en video, simulaciones clínicas, portafolios y evaluaciones de $360^{\circ}$, siendo en nuestro medio probablemente un sistema de evaluación más completo, pero difícil de desarrollar [8-10].

La evaluación de conocimientos sobre ventilación mecánica no es un fenómeno común, seguramente se da como un aprendizaje de tipo informal, al no estar considerado en todos los programas educativos y en otros de manera, quizás, muy tardía. Tampoco en nuestro medio existen instrumentos para evaluarlo.

El objetivo de nuestro estudio fue validar un instrumento en castellano sobre la evaluación de la ventilación mecánica para conocer el nivel de conocimientos en médicos residentes.

\section{Sujetos y métodos}

Se realizó una prueba piloto para validar un instrumento de evaluación sobre ventilación mecánica durante el periodo enero-julio de 2011. Se incluyeron médicos residentes en diferentes años de formación de las especialidades de anestesiología, medicina interna y urgencias médico-quirúrgicas del Hospital General Regional n. 46 'Lázaro Cárdenas' del Instituto Mexicano del Seguro Social, en Guadalajara, Jalisco, México.

El tipo de muestreo fue no probabilístico por conveniencia. El protocolo se sometió a aprobación por el Comité de Investigación y Ética en Salud del hospital. Todos los residentes aceptaron participar en el estudio de forma voluntaria previo consentimiento informado.

\section{Validación al castellano}

Se aplicó un instrumento de evaluación sobre ventilación mecánica, previamente validado en su idioma original en inglés; éste constaba de un examen estructurado con cuatro casos clínicos que incluyeron 19 preguntas de opción múltiple, evaluando áreas específicas sobre ventilación mecánica: fisiología cardiopulmonar, indicaciones de ventilación mecánica, diagnóstico y manejo de complicaciones asociadas al ventilador, uso de diferentes modos de 
ventilación e interpretación de datos en ventilación mecánica [11-13]. Posteriormente se tradujo del inglés al castellano, siguiendo el método descrito por Olivera-Cañadas et al [14]. La traducción fue realizada por dos personas bilingües con lengua castellana de origen, ajenos al estudio, pero con conocimientos médicos; posteriormente se realizó una discusión simultánea entre los traductores y el responsable de la investigación, de donde surgió un primer borrador del cuestionario. Se evaluó posteriormente por un médico traductor bilingüe, que tenía el inglés como lengua de origen, de donde surgió la versión de la prueba que se empleo. El examen se aplicó de forma individual y por grupos de especialidad, y no se fijó un tiempo límite para contestar el cuestionario (Anexo).

\section{Manejo de la información}

Se calificaron los cuestionarios de acuerdo con la plantilla del artículo original de donde se tomó el examen [11]. A cada inciso del cuestionario se le asignó un equivalente a las letras a, b, c, d. Se consideró que tenía los conocimientos en ventilación mecánica si contestaba de forma correcta las preguntas $1,2,3,6,11,12,18$ y 19 , siguiendo el modelo de Cox et al [11] (Tabla I).

Se consideró de forma individual una calificación mínima aprobatoria de 60 puntos y un nivel suficiente de conocimientos por encima de 80 . Se calificó de igual forma en grupos por especialidad y por año de residencia.

\section{Análisis estadístico}

Se efectuó por medio del programa estadístico SPSS v. 18.0. Se utilizó estadística descriptiva. Adicionalmente, se evaluó el grado de dificultad de cada pregunta dividiendo el número de veces que el reactivo fue contestado correctamente, entre el número total de alumnos a los que se propuso el reactivo en el examen, siendo el intervalo de calificación de 0 a 1 (0, más difícil; 1 , más fácil).

El índice de discriminación de cada pregunta fue la relación entre los alumnos que contestaron correctamente la pregunta y aprobaron el examen, entre aquellos que contestaron de manera incorrecta la pregunta y suspendieron el examen, de tal manera que si una pregunta con mayor índice de discriminación es contestada correctamente por un alumno existe mayor probabilidad de que el tema sea dominado por el alumno y apruebe el examen. Para evaluar este índice de discriminación se utilizó el índice de Pemberton [15], donde el intervalo de discrimina-
Tabla I. Número de pregunta y área de la ventilación mecánica que se evalúa de acuerdo con el cuestionario de Cox.

\begin{tabular}{ll}
\hline Pregunta 1 & Identificar paciente candidato a ventilación no invasiva \\
\hline Pregunta 2 & Diagnosticar la presencia de auto-PEEP \\
\hline Pregunta 3 & Manejo correcto de auto-PEEP \\
\hline Pregunta 6 & Identificar al paciente candidato a destete \\
\hline Pregunta 11 & Aplicación correcta de volumen corriente $(6 \mathrm{~mL} / \mathrm{kg})$ en pacientes con SIRPA \\
\hline Pregunta 12 & Uso apropiado de PEEP en un paciente con hipoxemia \\
\hline Pregunta 18 & Identificar el método correcto de medir auto-PEEP \\
\hline Pregunta 19 & Diagnóstico de neumotórax a tensión
\end{tabular}

PEEP: presión positiva al final de la espiración; SIRPA: síndrome de insuficiencia respiratoria pulmonar aguda.

Tabla II. Nivel de conocimientos del total de los residentes $(n=81)$.

\begin{tabular}{lc}
\hline No aprobados & $53(65 \%)$ \\
\hline Calificación mínima aprobatoria & $25(31 \%)$ \\
\hline Nivel de conocimientos suficiente & $3(4 \%)$ \\
\hline
\end{tabular}

ción utilizado fue de 0 a 0,23 , considerando 0 cuando la pregunta no tiene capacidad de discriminación y 0,23 cuando la pregunta más discrimina entre aquellos que dominan la materia y aquellos que no.

\section{Resultados}

Se aplicaron un total de 81 cuestionarios; de éstos, $37(46 \%)$ correspondieron a médicos residentes de urgencias médico-quirúrgicas, 30 (37\%) a médicos residentes de medicina interna y 14 (17\%) a residentes de anestesiología. La distribución de los residentes por año fue de la siguiente forma: primer año, 41 (51\%); segundo año, 17 (21\%); tercer año, 22 (27\%), y cuarto año, 1 (1\%). La calificación mínima obtenida fue de cuatro aciertos (21 puntos sobre 100) y la calificación máxima, de 17 aciertos $(90$ puntos sobre 100). Se obtuvo una media de $10 \pm 3$ aciertos (52 puntos), con una mediana también de 52 puntos. Del total de encuestados, el nivel de conocimientos fue insuficiente en la mayoría: sólo tres residentes de la muestra obtuvieron una califi- 
Tabla III. Resultados de calificación por año y por especialidad.

\begin{tabular}{|c|c|c|c|c|c|c|c|c|}
\hline & \multicolumn{2}{|c|}{ 1. er año $(n=41)$} & \multicolumn{2}{|c|}{ 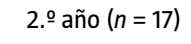 } & \multicolumn{2}{|c|}{ 3. er año $(n=22)$} & \multicolumn{2}{|c|}{ 4.ำ año $(n=1)$} \\
\hline & Puntos & $n$ & Puntos & $n$ & Puntos & $n$ & Puntos & $n$ \\
\hline Anestesiología & 48 & 10 & 57 & 4 & 0 & 0 & 0 & 0 \\
\hline Medicina interna & 48 & 18 & 50 & 3 & 55 & 8 & 68 & 1 \\
\hline $\begin{array}{l}\text { Urgencias médico- } \\
\text { quirúrgicas }\end{array}$ & 40 & 13 & 68 & 10 & 60 & 14 & 0 & 0 \\
\hline
\end{tabular}

Tabla IV. Grado de dificultad e índice de discriminación del cuestionario de Cox.

\begin{tabular}{lcc}
\hline & $\begin{array}{c}\text { Grado de dificultad }{ }^{\text {a }} \\
\text { (media: } 0,55)\end{array}$ & $\begin{array}{c}\text { Índice de discriminación }{ }^{b} \\
\text { (media: 0,06) }\end{array}$ \\
\hline $\begin{array}{l}\text { 1. Identifica paciente candidato a ventilación no invasiva } \\
\text { 2. Diagnostica la presencia de auto-PEEP }\end{array}$ & 0,60 & 0,04 \\
\hline $\begin{array}{l}\text { 3. Manejo correcto de auto-PEEP } \\
\text { 6. Identifica paciente candidato a destete }\end{array}$ & 0,64 & 0,04 \\
\hline $\begin{array}{l}\text { 11. Aplicación correcta de volumen corriente } \\
\text { a pacientes con SIRPA }\end{array}$ & 0,79 & 0,02 \\
\hline $\begin{array}{l}\text { 12. Uso apropiado de PEEP en un paciente con hipoxemia } \\
\text { 18. Identificar método correcto de medir auto-PEEP }\end{array}$ & 0,28 & 0,13 \\
\hline \begin{tabular}{l} 
19. Diagnóstico de neumotórax a tensión \\
\hline
\end{tabular} & 0,76 & 0,07 \\
\hline
\end{tabular}

PEEP: presión positiva al final de la espiración; SIRPA: síndrome de insuficiencia respiratoria pulmonar aguda. a Se obtiene dividiendo el número de veces que el reactivo fue contestado correctamente, entre el número total de alumnos a los que se propuso el reactivo en el examen, siendo el intervalo de calificación de 0 a 1 ( 0 , más difícil; 1, más fácil). ' Es la relación de los alumnos que contestaron correctamente la pregunta y aprobaron el examen, entre aquellos que contestaron de manera incorrecta la pregunta y suspendieron el examen; el intervalo de discriminación utilizado fue de 0 a 0,23, considerando 0 cuando la pregunta no tiene capacidad de discriminación y 0,23 si la pregunta es la que más discrimina entre aquellos que dominan la materia y aquellos que no.

cación de suficiente para el manejo de la ventilación mecánica (Tabla II).

En la tabla III se muestran las calificaciones por año y especialidad de los residentes evaluados.

En la tabla IV se refleja el grado de dificultad de los ocho ítems que evaluaron conocimientos en ventilación mecánica y su índice de discriminación. El rango del grado de dificultad encontrado fue de 0,28 a 0,79 y el rango del índice de discriminación fue de 0 a 0,135; la pregunta con mayor índice de discriminación se catalogó como de dificultad intermedia y la pregunta que no discriminó fue la segunda más fácil.
En los resultados, el 65\% de los residentes obtuvieron una calificación menor de 60 puntos, concluyendo que el instrumento de evaluación tiene una dificultad alta.

De acuerdo con lo anterior, las preguntas con mayor grado de dificultad fueron las que tuvieron menor puntuación, correspondiendo a las preguntas 2,11 y 12 .

La pregunta 12 fue contestada correctamente por 23 (28\%) médicos residentes evaluados. En total, sólo 11 (48\%) de los 23 residentes que contestaron bien esta pregunta aprobaron el examen. La pregunta 3 fue contestada correctamente por 36 (44\%) residentes de la muestra total. En total, 16 (44\%) de esos 36 residentes aprobaron el examen. La pregunta 11 fue contestada de forma correcta por 37 (46\%) del total de los médicos evaluados, y sólo 21 (56\%) de ellos aprobaron el examen.

De manera secundaria se evaluó el índice de discriminación y el grado de dificultad de las preguntas que no se tomaron en cuenta para la evaluación de alguna área específica de conocimientos sobre ventilación mecánica en el instrumento original en inglés, con el fin de determinar su utilidad. Se encontró en la mayoría un grado de dificultad intermedio. Sólo la pregunta 14 (que se refiere a los conocimientos de fisiología pulmonar) tuvo un grado alto de dificultad, pero fue la pregunta con menor grado de discriminación. Las dos preguntas con mayor índice de discriminación fueron la 7 y la 17 (Tabla V).

En cuanto a la pregunta catalogada como la más difícil (n. $\left.{ }^{\circ} 14\right)$, fue contestada correctamente por 14 (17\%) de los médicos. Sólo 4 (28\%) de éstos aprobaron el examen.

La pregunta con mayor grado de discriminación fue la 17: este ítem fue contestado correctamente por 33 (41\%) médicos, de los cuales sólo 13 (39\%) aprobaron el examen. Al realizar una evaluación de conocimientos de forma global, considerando todos los residentes de todas las especialidades, se detectaron las mayores deficiencias de conocimientos en las preguntas sobre el manejo correcto de autoPEEP (presión positiva al final de la espiración), uso apropiado de PEEP en pacientes con hipoxemia, aplicación correcta de volumen corriente en el síndrome de insuficiencia pulmonar aguda (SIRPA) y diagnóstico de neumotórax a tensión (Tabla VI).

\section{Discusión}

Este es el primer estudio en nuestro medio donde se valida un instrumento al castellano para conocer el nivel de conocimientos sobre ventilación mecánica 
en estudiantes de posgrado a través de un instrumento previamente validado en inglés. La falta de evaluación y los instrumentos adecuados siguen siendo una debilidad de nuestros programas educativos. Nuestro hospital es la unidad de atención médica más grande de segundo nivel de atención médica del Occidente de México, con 423 camas. En él se desarrollan diez especialidades, tanto médicas como quirúrgicas, con una plantilla actual de 112 residentes. Al ser un hospital de segundo nivel sólo se atienden pacientes cuya complejidad de resolución no requiere procedimientos invasivos, como cateterismo cardiaco, trasplante de órganos o procedimientos microquirúrgicos avanzados. De ahí que, en promedio, el $50 \%$ de sus residentes realizan sólo su primer año en el hospital, y el resto de su residencia, en los hospitales de concentración o tercer nivel, lo que explica la mayor participación de residentes de primer año en nuestro estudio. Otro punto relevante en la muestra fue la negativa a participar de los residentes de cuarto año disponibles en nuestra unidad, seguramente por el temor a ser evaluados, a pesar de ser una evaluación anónima.

Actualmente, en México, como en otras latitudes, existe una carencia notable de médicos especialistas, particularmente en las áreas de medicina interna y cuidados intensivos. Esto obliga a que los médicos residentes asuman estas funciones, incluso casi desde el inicio de su residencia, sin tener en cuenta la complejidad que estos pacientes representan y los múltiples parámetros que se deben dominar en esta área en especial $[16,17]$.

En nuestro estudio encontramos que el $65 \%$ de los residentes evaluados no tienen un nivel de conocimientos aceptable sobre ventilación mecánica, y sólo el $4 \%$ de los residentes se consideraron aptos para el uso y manejo de la ventilación mecánica según el instrumento aplicado. Este número es mínimo para la cantidad de pacientes intubados que se encuentran en los diferentes servicios fuera de la unidad de cuidados intensivos en nuestro hospital, pero puede ser reflejo de lo que sucede en otras regiones.

$\mathrm{Al}$ analizar los resultados por grado y año de especialidad se encontró que los residentes de medicina interna y anestesiología tenían calificaciones ligeramente superiores a los residentes de urgencias médico-quirúrgicas, particularmente en el primer año. Sin embargo, los residentes de urgencias médico-quirúrgicas de segundo año obtuvieron calificaciones muy superiores a los residentes de medicina interna y anestesiología, y esto puede deberse a que los residentes de esta especialidad llevan un módulo educativo sobre ventilación mecánica en el segundo año. Esta misma explicación podría aplicarse
Tabla V. Grado de dificultad e índice de discriminación del cuestionario de Cox de las preguntas que no evaluaron el grado de conocimientos.

\begin{tabular}{lcc}
\hline & Grado de dificultad $^{\mathrm{a}}$ & Índice de discriminación \\
\hline Pregunta 4 & 0,33 & 0,09 \\
\hline Pregunta 5 & 0,35 & 0,07 \\
\hline Pregunta 7 & 0,59 & 0,12 \\
\hline Pregunta 8 & 0,55 & 0,09 \\
\hline Pregunta 9 & 0,60 & 0,09 \\
\hline Pregunta 10 & 0,56 & 0,07 \\
\hline Pregunta 13 & 0,33 & 0,02 \\
\hline Pregunta 14 & 0,17 & 0,04 \\
\hline Pregunta 15 & 0,72 & 0,06 \\
\hline Pregunta 16 & 0,40 & 0,06 \\
\hline Pregunta 17 & 0,35 & 0,16 \\
\hline
\end{tabular}

a,b Véase el pie de la tabla IV.

al hecho de que los residentes de medicina interna de tercer y cuarto año obtienen promedios más altos, al tener sus rotaciones por la unidad de cuidados intensivos.

En el estudio de Cox et al [11], las puntuaciones más altas se asociaron a una adecuada percepción de los conocimientos sobre ventilación mecánica y a una claridad en los objetivos de aprendizaje. Sin embargo, no se encontró una relación de mayores puntuaciones en médicos rotatorios a la unidad de cuidados intensivos ni en aquellos que tuvieron planes de realizar esta especialidad.

En la evaluación de áreas específicas de la ventilación mecánica, los residentes que participaron en el estudio tienen conocimientos para diagnosticar y medir el auto-PEEP, pero al momento de utilizarlo en la práctica cotidiana, sólo el $44 \%$ sabe emplearlo de forma adecuada. El estudio de Cox et al demostró resultados similares. Consideramos que este fenómeno se debe a que el residente no ha desarrollado su aptitud clínica para la aplicación de los conocimientos obtenidos de la bibliografía o lo aprende de manera informal posteriormente. Datos similares se reflejan en la aplicación de PEEP en un paciente con hipoxemia y el diagnóstico de neumotórax como complicación de ventilación mecánica, 
Tabla VI. Número de médicos que contestó las diferentes preguntas correctamente por año y por especialidad.

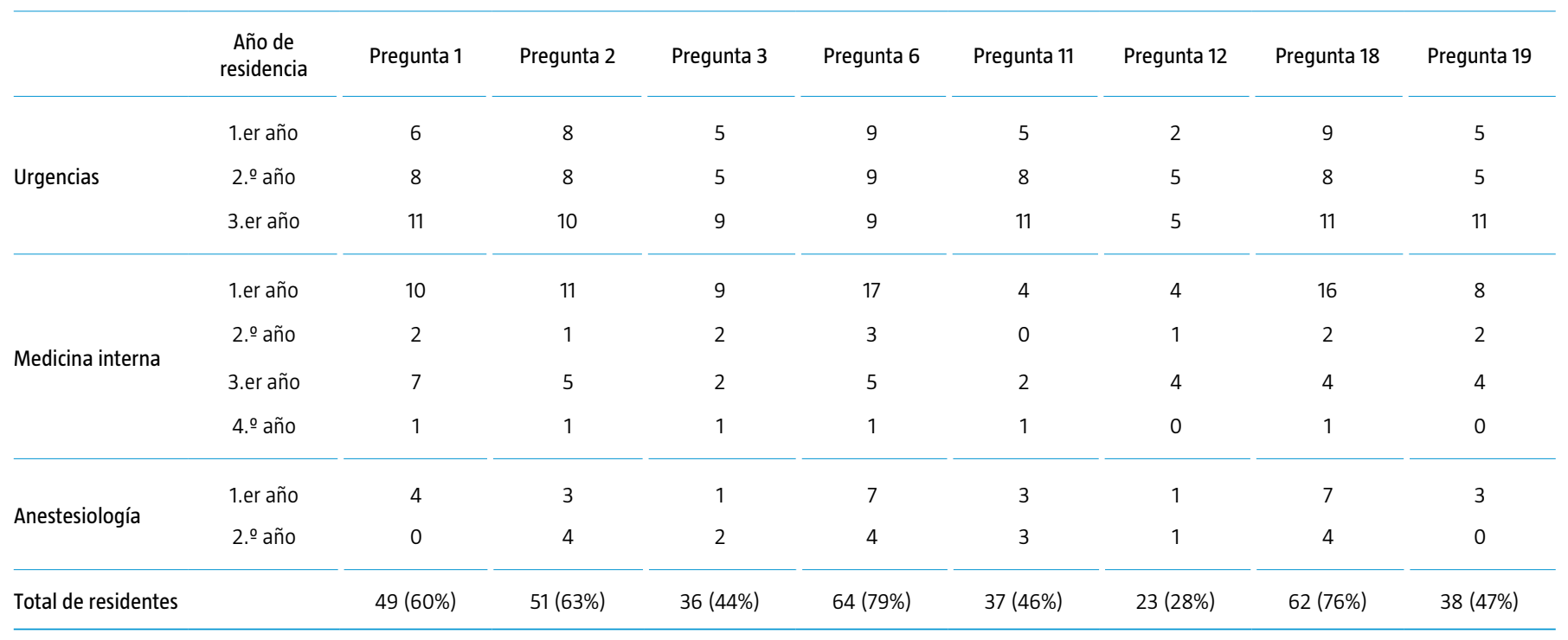

contestado de forma correcta sólo por el $28 \%$ y $47 \%$ de los médicos, respectivamente.

En el mismo estudio descrito por Cox et al se asocia la aplicación correcta de volumen corriente bajo, la identificación del paciente apto para destete y la identificación del paciente para ventilación no invasiva, como parámetros que si se aplican de forma correcta, reducen los costes hospitalarios y disminuyen la mortalidad en los pacientes. En nuestra muestra hallamos calificaciones bajas en estas áreas, excepto en reconocer al paciente candidato a destete, por lo que también deducimos que esto puede estar reflejándose en la estancia hospitalaria y la morbimortalidad de los pacientes.

Se ha demostrado que el paciente sometido a ventilación mecánica puede responder de manera adecuada al manejo del ventilador hasta en un 35\%, lo que se traduce en una disminución del periodo de intubación y de días de estancia [17].

Evaluamos las preguntas con mayor grado de dificultad y encontramos tres reactivos: la pregunta 12, que se relaciona con el uso de auto-PEEP, sólo la contestó acertadamente el 48\%. La pregunta 3, sobre el manejo de auto-PEEP, sólo el $44 \%$, y la pregunta 11, sobre el volumen corriente en el SIRPA, sólo el $54 \%$. Si bien no podemos afirmar que fueron contestadas por efecto del azar, tampoco podemos afirmar que se debe a que el médico residente tenía una mejor percepción sobre el tema. En nuestro estudio, el 79\% de los residentes fue capaz de contestar acertadamente la pregunta 6 , la cual identifica al paciente candidato a destete de ventilador, pero el índice de discriminación fue muy bajo $(0,02)$, lo que significa que la mayoría de los residentes que contestaron adecuadamente esta pregunta no aprobaron el examen, por lo que no podemos concluir al respecto del dominio que se tiene acerca del tema por parte del residente.

La fiabilidad de un instrumento para evaluar los conocimientos es determinada por el número de elementos incluidos en la prueba, el grado de discriminación de cada elemento, si existen preguntas que evalúen puntos específicos acerca de un tema, y el grado de dificultad de la prueba.

Una de las debilidades de este instrumento es que sólo dispone de 19 ítems, lo que por probabilidad, según la tabla de Ebel, le otorga menos del 50\% de fiabilidad [15]. Sin embargo, para nuestra población, la fortaleza del cuestionario es que evalúa puntos específicos de ventilación mecánica y su grado de dificultad, además de que la media y la mediana de los resultados son iguales (52 puntos), lo que fortalece la homogeneidad del grupo. Ello incrementa la fiabilidad del estudio a pesar del número de elementos del cuestionario. Al realizar la validación de todas las preguntas, encontramos diversidad en el grado de dificultad de todas ellas, incluyendo las que no se toman en cuenta para la evaluación del alumno. Consideramos que esta versión en castellano es aplicable y útil como instrumento de evaluación de conocimientos en ventilación mecánica a estudiantes de posgrado, en particular aque- 
llos que ya han llevado en su programa esta temática o han efectuado rotaciones en áreas donde hay pacientes con soporte ventilatorio.

Estamos de acuerdo en que el aprendizaje debe basarse en la recuperación de las experiencias, de la revisión diaria de pacientes que permita el desarrollo de la aptitud clínica. El desarrollo de esta aptitud se basa en casos reales por medio de la observación clínica, el análisis documental y la crítica de evidencias [18].

Con estos resultados consideramos que es imperativo adecuar al plan de estudios o currículo de cada especialidad un módulo de ventilación mecánica, en particular, aquellas residencias donde el médico en formación deba atender a este tipo de pacientes desde el ingreso de su especialidad. En diversos estudios [19-21] se evaluaron aptitudes clínicas de médicos residentes sobre manejo del traumatismo craneoencefálico, competencias profesionales y sangrados en la segunda mitad del embarazo, en los cuales obtuvieron resultados similares al nuestro, es decir, residentes con conocimientos deficientes en las áreas evaluadas.

Zaman [22] propuso un tutorial para elevar el nivel de conocimientos de ventilación mecánica en los residentes previamente a la rotación por la unidad de cuidados intensivos, que comprende un programa educativo sobre ondas de mecánica respiratoria, discusión de la mecánica respiratoria, el reconocimiento y manejo de las complicaciones relacionadas con la ventilación mecánica y conceptos sobre la configuración de modos convencionales de ventilación. En su estudio, con resultados similares al nuestro, hubo una mejoría del nivel de conocimientos al aplicar su programa educativo, comunicando un pretest de 5,1 y un postest de 6,4 , con una reducción de la desviación estándar de 1,6 a 1,2. De igual forma, otros autores afirman la necesidad de aplicar una enseñanza que favorezca el pensamiento reflexivo y crítico de los médicos en formación, lo que repercutiría en su desarrollo personal y profesional y en la atención de los pacientes [23].

Consideramos que el instrumento utilizado en su versión al castellano cumple con los objetivos de evaluación, es fiable, fácilmente entendible, abarca los aspectos más relevantes de la ventilación mecánica y es de mediana a alta complejidad, por lo que puede estandarizarse su uso y aplicación.

\section{Bibliografía}

1. Reta-De Rosas AM, López MJ, Vargas AL, Montbrun MC.
Evaluación de competencias médicas en un currículo de grado no diseñado por competencias. Educ Med 2008; 11: 207-18.

2. Ruelas B. Una nueva era de la certificación de hospitales en México. Cir Cir 2010; 78: 201-2.

3. Chisholm A, Askham J. What do you think of your doctor? A review of questionnaires for gathering patients' feedback on their doctor. Picker Institute Europe; 2006. URL: http:// www.engage.hscni.net/library/What\%20do\%20you\%20 think.pdfhttp://www.engage.hscni.net/library/What\%20 do\%20you\%20think.pdf. [12.01.2012].

4. Cumplido-Hernández G. Visión pragmática de la educación médica. Rev Med Inst Seguro Soc 2009; 47: 171-8.

5. Weinberger SE, Smith LG, Collier VU. Redesigning training for internal medicine. Ann Intern Med 2006; 144: 927-32.

6. Marple BF. Competency-based resident education. Otolaryngol Clin N Am 2007; 40: 1215-25.

7. Charlson ME, Karnik J, Wong M, McCulloch CE, Hollenberg JP. Does experience matter? A comparison of the practice of attendings and residents. J Gen Intern Med 2005; 20: 497-503.

8. Epstein RM. Assessment in medical education. N Engl J Med 2007; 356: 387-96.

9. Smee S. Skill based assessment. BMJ 2003; 326: 703-6.

10. Blackburn GL. Teaching, learning, doing: best practices in education. Am J Clin Nutr 2005; 82 (Suppl 1): S218-21.

11. Cox CE, Carson SS, Ely EW, Govert JA, Garret JM, Brower RG, et al. Effectiveness of medical resident education in mechanical ventilation. Am J Respir Crit Care Med 2003; 167: 32-8.

12. Alvarado A. Ventilación mecánica (revisión bibliográfica). Rev Med Costa Rica 2008; 584: 181-209.

13. Camacho V, coord. Síndrome de insuficiencia respiratoria pulmonar aguda SIRPA. Rev Cub Med Int Emerg 2004; 3: $17-51$.

14. Olivera-Cañadas G. Silvestre-Busto C, Lorenzo-Martínez S, Astier-Peña MP, Torijano-Casalengua ML, MaderueloFernández JA. Traducción y validación de la encuesta de la AHRQ para medir la cultura de la seguridad del paciente en atención primaria; 2009. URL: http://www.seguridaddelpaciente.es/contenidos/castellano/poster_encuesta_AHRQ_ primaria_Granada 2009.pdf?phpMyAdmin $=$ mvRYxVABNPM34i7Fnm\%2C23Wrlq5. [20.01.2012].

15. Córica J, Hernández M, Propuesta de indicadores de calidad para la evaluación de la calidad de las preguntas en los exámenes de opción múltiple. I Congreso Virtual Latinoamericano de Educación a Distancia; 2004. URL: http://www.ateneonline. net/datos/91_03\%20ponencia\%20corica-hernandez-hollowaydimou.pdf. [05.01.2012].

16. Narro-Robles J, Ruiz-Ruisánchez A. El papel del médico general en la atención médica en el México actual. Gac Med Mex 2004; 140: S13-20.

17. Schmidt M, Demoule A, Polito A, Porchet R, Aboab J, Siami S, et al. Dyspnea in mechanically ventilated critically ill patients. Crit Care Med 2011; 39: 2059-65.

18. Chavarría RA, Rivera DB, Entorno laboral y aptitudes clínicas en residentes de urgencias médico-quirúrgicas. Rev Med Inst Mex Seguro Soc 2004; 42: 371-8.

19. Arredondo E, Castillo E, Sánchez F, La aptitud clínica de los residentes de ortopedia y traumatología en el manejo del paciente politraumatizado (dependiendo del mecanismo de la lesión). Acta Ortop Mex 2006; 20: 64-71.

20. Rocha M, San Juan MA, Evaluación de la competencia profesional de residentes de medicina general integral para la atención a la familia. Educ Med 2010; 13: 41-6.

21. Veloz MG, Uribe J, Lemus SR, Aptitud clínica de médicos residentes en el tratamiento de sangrados de la segunda mitad del embarazo. Rev Med Inst Mex Seguro Soc 2007; 45: 459-62.

22. Zaman MU. Enhancing competence of mechanical ventilation skills among medical residents: an innovative approach. Chest 2009; 136: 115.

23. Rocha JM, Loria J, Aptitudes clínicas de residentes de urgencias en el abordaje de la enfermedad vascular cerebral. Educ Med Super 2007; 21: 1 . 
Anexo. Cuestionario de evaluación sobre conocimientos en

el manejo de ventilación mecánica en médicos residentes.

\section{Caso 1}

Una mujer de 64 años con antecedentes de enfermedad pulmonar obstructiva crónica se presenta en urgencias con dificultad respiratoria en aumento. Usa broncodilatadores hasta cuatro veces al día. Durante la semana pasada, su tolerancia al ejercicio se ha ido reduciendo y ahora presenta disnea en reposo a pesar del empleo frecuente de sus $\beta$-agonistas. La paciente muestra tos moderada y esputo hialino. En el examen físico, tiene dificultad respiratoria moderada, pero se encuentra alerta y responde a estímulos. Temperatura corporal de $38{ }^{\circ} \mathrm{C}$, frecuencia cardiaca de 110 latidos/min, frecuencia respiratoria de 28 respiraciones/min, presión arterial de 110/70 $\mathrm{mmHg}$. La paciente usa los músculos accesorios para respirar, pero puede completar frases u oraciones cortas. Se encuentra disminución de los ruidos respiratorios bilaterales y se nota espiración prolongada. Los ruidos cardiacos son distantes, pero regulares, y no hay relevancia en la exploración física del abdomen. Una radiografía de tórax objetiva hiperinsuflación pulmonar y disminución del contorno del pulmón a lo largo de ambos campos pulmonares. Una gasometría arterial realizada en la paciente con oxígeno a $2 \mathrm{~L} / \mathrm{min}$ con puntas nasales revela un $\mathrm{pH}$ de $7,30, \mathrm{PaCO}_{2}$ de $60 \mathrm{mmHg}, \mathrm{PaO}_{2}$ de $58 \mathrm{mmHg}$ y $\mathrm{SaO}_{2}$ del $88 \%$.

1. Además de una cuidadosa vigilancia, ¿cuál de las siguientes intervenciones sería la más apropiada en este momento?:

a) Aumentar el oxígeno suplementario además de la terapia continua con $\beta$-agonistas.

b) Sólo la terapia continua con $\beta$-agonistas.

c) Intubación y ventilación mecánica.

d) Ventilación no invasiva con presión positiva (BIPAP).

Durante el curso de su tratamiento, se toma la decisión de intubar a la paciente. Después de un bolo de líquidos de $500 \mathrm{~mL}$, la paciente es sedada e intubada con tubo endotraqueal $n .{ }^{\circ} 7$, que se coloca $\sin$ dificultad. El indicador de $\mathrm{CO}_{2}$ muestra la adecuada colocación del tubo endotraqueal. La paciente es ventilada a 25 respiraciones/min con dispositivo válvula bolsa mascarilla (AMBU). Sin embargo, inmediatamente después de la intubación se observa una presión arterial de 70/40 $\mathrm{mmHg}$.
2. ¿Cuál es la causa mas probable de la hipotensión?:

a) Sepsis.

b) Neumotórax.

c) Aumento de la presión intratorácica (auto-PEEP).

d) Infarto agudo al miocardio.

3. La intervención inicial más apropiada para mejorar la afectación cardiovascular sería:

a) Aumentar los líquidos por vía intravenosa.

b) Colocar una aguja de calibre 16 en el segundo espacio intercostal izquierdo línea media anterior.

c) Dejar de usar AMBU-bolsa y permitir que la paciente exhale.

d) Empezar con $5 \mu \mathrm{g} / \mathrm{kg} / \mathrm{min}$ de dopamina.

4. La presión arterial mejora a 95/65 $\mathrm{mmHg}$. ¿Cuál de los siguientes parámetros del ventilador sería el más adecuado para esta paciente $(60 \mathrm{~kg})$ ?:

a) Asisto controlado por volumen, con frecuencia respiratoria de 22, volumen corriente de $400 \mathrm{~mL}$, PEEP de $5 \mathrm{cmH}_{2} \mathrm{O}$ y $\mathrm{FiO}_{2}$ del $100 \%$.

b) Asisto controlado por volumen, con frecuencia respiratoria de 12 , volumen corriente de $500 \mathrm{~mL}$, PEEP de $5 \mathrm{cmH}_{2} \mathrm{O}$ y $\mathrm{FiO}_{2}$ del $60 \%$.

c) Asisto controlado por volumen, con frecuencia respiratoria de 20, volumen corriente de $700 \mathrm{~mL}$, PEEP de $5 \mathrm{cmH}_{2} \mathrm{O}$ y $\mathrm{FiO}_{2}$ del $60 \%$.

d) Asisto controlado por presión, con frecuencia respiratoria de 15 , presión inspiratoria de $25 \mathrm{cmH}_{2} \mathrm{O}$, relación inspiración espiración de 1 a 1 , PEEP de $5 \mathrm{cmH}_{2} \mathrm{O}$ y $\mathrm{FiO}_{2}$ del $100 \%$.

5. En el modo asisto controlado por volumen (no regulado por presión), si la frecuencia respiratoria es de 16 respiraciones/min y el volumen corriente es de $600 \mathrm{~mL}$, ¿cuál de las siguientes aseveraciones resulta correcta acerca del volumen corriente aplicado si la frecuencia respiratoria del paciente es de 22 respiraciones/min?:

a) $600 \mathrm{~mL}$ cada respiración.

b) $600 \mathrm{~mL}$ durante las 16 respiraciones determinadas y el resto determinado por el esfuerzo del paciente.

c) El volumen corriente será determinado por el esfuerzo del paciente cada vez que respira. 
d) El volumen corriente puede variar, dependiendo de la función de distensibilidad pulmonar.

Después de 3 días de ventilación mecánica, la paciente se encuentra despierta, sigue las órdenes y tiene un adecuado reflejo de tos. Es colocada en presión positiva continua de la vía aérea (CPAP) de $5 \mathrm{cmH}_{2} \mathrm{O}$. Después de un minuto, tiene una frecuencia respiratoria de 20 por minuto y un volumen corriente de $300 \mathrm{~mL}$.

\section{De las siguientes opciones, ¿cuál sería el plan más adecuado para el día con respecto a la ventilación mecánica?:}

a) Sedar a la paciente y reanudar con ventilación asisto controlada por volumen.

b) Sedar a la paciente e iniciar con ventilación asisto controlada por presión.

c) Iniciar un método de destete de ventilación con presión de soporte o una pieza en $\mathrm{T}$.

d) Recomendar que la paciente sea evaluada para una traqueostomía.

\section{Caso 2}

Una mujer de 29 años (60 kg) es encontrada inconsciente en un parque de la ciudad después de ingerir una sustancia desconocida. La paciente es reanimada e intubada en el lugar por servicios médicos de emergencias. A su llegada a urgencias, la temperatura de la paciente es de de $38,4{ }^{\circ} \mathrm{C}$, la frecuencia cardiaca es de 110 latidos/min, y la tensión arterial, de 130/78 mmHg. Se succiona esputo espeso por el tubo endotraqueal. La exploración pulmonar revela crepitaciones en la base derecha, sin sibilancias. Los ruidos cardiacos son normales y la producción de orina es la adecuada. Una radiografía de tórax muestra un infiltrado denso en el lóbulo inferior derecho.

Se coloca a la paciente en ventilación mecánica sincronizada intermitente (SIMV) con volumen corriente de $450 \mathrm{~mL}$, frecuencia respiratoria de 16 respiraciones/min, PEEP de $5 \mathrm{cmH}_{2} \mathrm{O}$ y $\mathrm{FiO}_{2}$ del $40 \%$. Con estos parámetros la paciente registra una frecuencia respiratoria de 30 respiraciones/min. Una gasometría arterial muestra un $\mathrm{pH}$ de 7,45, $\mathrm{PaCO}_{2}$ de $34 \mathrm{mmHg}, \mathrm{PaO}_{2}$ de 50 mmHg y $\mathrm{SaO}_{2}$ del $83 \%$. $\mathrm{La} \mathrm{FiO}_{2}$ se aumenta al $100 \%$ y una gasometría arterial 30 minutos más tarde muestra un $\mathrm{pH}$ de 7,43, $\mathrm{PaCO}_{2}$ de $36 \mathrm{mmHg}, \mathrm{PaO}_{2}$ de $55 \mathrm{mmHg}$, y $\mathrm{SaO}_{2}$ del 89\%. La frecuencia respiratoria del paciente no se modifica.

\section{La principal anormalidad fisiológica responsable de la hipoxemia es:}

a) Ventilación excesiva del espacio muerto.

b) Bajo gasto cardiaco.

c) Corto circuito intrapulmonar.

d) Hipoventilación.

\section{8. ¿Cuál de las siguientes aseveraciones es correcta acerca de la SIMV?:}

a) El volumen corriente de respiraciones iniciado por el paciente, por encima de la tasa establecida, se determina por el total del volumen corriente del paciente.

b) El volumen corriente de respiraciones iniciado por el paciente, por encima de la tasa establecida, se determina por el esfuerzo del paciente.

c) El volumen corriente de cada respiración es determinado por el esfuerzo del paciente.

d) SIMV es una forma de ventilación ciclada por presión.

Más tarde, esa noche, una enfermera lo llama para examinar al paciente debido a que su saturación de $\mathrm{O}_{2}$ se ha reducido en los últimos cinco minutos del $94 \%$ al $80 \%$, a pesar de un aumento de la $\mathrm{FiO}_{2}$ a 1,0 (oxígeno al $100 \%$ ). En la exploración física se encuentra que los ruidos respiratorios son audibles bilateralmente, pero han disminuido de forma simétrica, y no hay presencia de sibilancias. La presión pico de la vía aérea se ha incrementado a $65 \mathrm{cmH}_{2} \mathrm{O}$ (de $40 \mathrm{cmH}_{2} \mathrm{O}$ ), pero la presión meseta está relativamente sin cambios a $25 \mathrm{cmH}_{2} \mathrm{O}$. Su frecuencia respiratoria es ahora de 42 respiraciones/min, su frecuencia cardiaca es de 110 latidos/min, y su presión arterial, de 150/85 mmHg.

\section{9. ¿Qué intervención sería la más probable para mejorar el proceso fisiológico que está causando la hipoxemia del paciente?:}

a) Descompresión de urgencia con aguja del supuesto neumotórax.

b) Administración urgente de activador tisular del plasminógeno, seguido de heparina.

c) Aspiración, seguida de cambio del tubo endotraqueal si no hay mejoría.

d) Broncodilatadores, seguido de metilprednisolona intravenosa.

Tras realizar una intervención adecuada, la paciente se estabiliza. Poco a poco, durante los próximos tres días, su oxigenación mejora gradualmente. La pacien- 
te se cambia a ventilación con presión soporte, con presión inspiratoria de $20 \mathrm{cmH}_{2} \mathrm{O}$ y PEEP de $5 \mathrm{cmH}_{2} \mathrm{O}$.

\section{0. ¿Cuál de los siguientes enunciados respecto al estado de las vías respiratorias del paciente en ventilación con presión soporte es cierto?:}

a) Los volúmenes corrientes serán los mismos con cada respiración.

b) El volumen minuto deberá ser constante.

c) El volumen minuto varía de acuerdo a su fuerza y esfuerzo respiratorio.

d) La relación inspiración/espiración es fijada por el médico.

\section{Caso 3}

Una mujer de 56 años con diabetes mellitus es llevada a la sala de urgencias con antecedente de tres días con disuria y dolor en la espalda baja. En urgencias no está completamente consciente y presenta taquipnea. Temperatura corproal de $38,9{ }^{\circ} \mathrm{C}$, frecuencia respiratoria de 28 respiraciones/min, frecuencia cardiaca de 120 latidos/min, tensión arterial de $80 / 50 \mathrm{mmHg}$ y peso ideal de $60 \mathrm{~kg}$. La paciente presenta mucosas secas y llenado capilar rápido. En la exploración física se encuentran ruidos inspiratorios con estertores débiles bilaterales, y el examen cardiaco es significativo sólo para la taquicardia. El examen abdominal es normal. No tiene edema pedio. El recuento de leucocitos es de $13.000 / \mathrm{mL}$ y el análisis de orina revela $>100.000$ leucocitos con abundantes bacterias. Una radiografía de tórax muestra infiltrados intersticiales bilaterales sin derrames. Una gasometría arterial tomada mientras el paciente respira con mascarilla y oxígeno al $100 \%$ revela un $\mathrm{pH}$ de 7,30, $\mathrm{PaCO}_{2}$ de $25 \mathrm{mmHg}, \mathrm{PaO}_{2}$ de $62 \mathrm{mmHg}$ y $\mathrm{SaO}_{2}$ del $90 \%$. Mientras se inicia tratamiento con líquidos y antibióticos por vía intravenosa, se considera que el soporte ventilatorio está indicado.

\section{1. ¿Cuál de las siguientes opciones sería la forma más apropiada de soporte ventilatorio en este momento?}

a) BIPAP con mascarilla facial.

b) Intubación y ventilación asisto controlada por volumen, frecuencia respiratoria de 20 respiraciones/min, volumen corriente de $360 \mathrm{~mL}$.

c) Intubación y ventilación mandatoria intermitente, frecuencia respiratoria de 20 respiraciones/min, volumen corriente de $700 \mathrm{~mL}$.

d) Intubación y ventilación asisto controlada por volumen, frecuencia respiratoria de 20 respiraciones/min, volumen corriente de $700 \mathrm{~mL}$.

En el segundo día de estancia hospitalaria se está manejando con ventilación asisto controlada por volumen. La presión arterial es de 100/70 mmHg, y la frecuencia cardiaca, de 90 latidos/min. Mientras la paciente está sedada, su presión pico es de $40 \mathrm{cmH}_{2} \mathrm{O}$, con una $\mathrm{FiO}_{2}$ del $60 \%$ y PEEP de $5 \mathrm{cmH}_{2} \mathrm{O}$. La gasometría arterial revela un $\mathrm{pH}$ de $7,28, \mathrm{PaCO}_{2}$ de $36 \mathrm{mmHg}$ y $\mathrm{PaO}_{2}$ del $85 \%$.

\section{2. ¿Cuál de las siguientes opciones sería la medida más apropiada a seguir?:}

a) Aumentar la $\mathrm{FiO}_{2}$ al $80 \%$.

b) Aumentar el volumen corriente a $100 \mathrm{~mL}$ de su valor inicial.

c) Aumentar la frecuencia respiratoria 4 respiraciones/min de los parámetros iniciales.

d) Aumentar PEEP a $10 \mathrm{cmH}_{2} \mathrm{O}$.

\section{3. ¿Cuál de las siguientes intervenciones} prolongaría el tiempo espiratorio de la paciente que recibe ventilación asisto controlada por volumen?:

a) Aumentar la frecuencia respiratoria.

b) Aumentar el flujo inspiratorio.

c) Aumentar PEEP.

d) Aumentar el volumen corriente.

Después de varios días, la paciente estaba siendo tratada con ventilación asisto controlada por presión, con una frecuencia de 18 respiraciones/min, PEEP de $12 \mathrm{cmH}_{2} \mathrm{O}$ y presión inspiratoria total de $30 \mathrm{cmH}_{2} \mathrm{O}$. La relación inspiración/espiración es de 1 a 1 . Inicialmente, con esos parámetros, la medición de volumen corriente obtenido da un promedio de $400 \mathrm{~mL}$. Dos días después, ahora con los mismos parámetros, el volumen corriente obtenido es de $500 \mathrm{~mL}$.

14. ¿Cuál de las siguientes circunstancias es la más probable para explicar el cambio del volumen corriente?

a) Aumento en el esfuerzo respiratorio por el paciente.

b) Aumento de la distensibilidad pulmonar.

c) Aumento de la ventilación del espacio muerto.

d) Atrapamiento de aire que conduce a aumentar la PEEP intrínseca (auto-PEEP).

En el decimocuarto día de estancia intrahospitalaria, la paciente está afebril, hemodinámicamente es- 
table y tiene una presión venosa central de $12 \mathrm{mmHg}$. Ha sido tratada con ventilación asisto controlada por volumen los últimos siete días, y actualmente recibe un volumen corriente de $360 \mathrm{~mL}$, con frecuencia de 24 respiraciones/min, PEEP de $15 \mathrm{cmH}_{2} \mathrm{O}$ y $\mathrm{FiO}_{2}$ del 50\%. Una gasometría arterial en este momento revela un $\mathrm{pH}$ de $7,35, \mathrm{PaCO}_{2}$ de $50 \mathrm{mmHg}$, $\mathrm{PaO}_{2}$ de 90 mmHg y $\mathrm{SaO}_{2}$ del $97 \%$, además de $\mathrm{HCO}_{3}$ sanguíneo de $27 \mathrm{mEq} / \mathrm{L}$, presión pico de $48 \mathrm{cmH}_{2} \mathrm{O}$ y presión meseta de $38 \mathrm{cmH}_{2} \mathrm{O}$.

\section{5. ¿Cuál de las siguientes opciones sería la intervención más apropiada en este momento?:}
a) Disminución de la PEEP.
b) Iniciar infusión de bicarbonato de sodio.
c) Aumentar el volumen corriente.
d) Dar furosemida por vía intravenosa.

En el vigesimoprimer día de estancia intrahospitalaria se realiza una traqueostomía en la camilla de la paciente, sin complicaciones. Esa noche, tras ser volteada durante el baño, suenan las alarmas de presión pico en el ventilador y se observa en el monitor una disminución de la $\mathrm{SaO}_{2}$. Al examinarlo, el paciente tiene dificultad respiratoria y taquicardia. El tubo de traqueostomía aparece desplazado de su posición original. El examen pulmonar revela ruidos respiratorios disminuidos bilateralmente, sin sibilancias. El cuello de la paciente se observa hinchado y la piel del cuello y tórax anterior crepita al tacto.

\section{6. ¿Cuál de las siguientes es la intervención inmediata más apropiada?:}

a) Pedir una radiografía portátil de tórax.

b) Colocar un tubo pleural en el tórax bilateral.

c) Intentar reemplazar el tubo de traqueostomía.

d) Retirar el tubo de traqueostomía y colocar un tubo endotraqueal.

\section{Caso 4}

Una mujer de 35 años (60 kg) con asma es intubada al llegar al servicio de urgencias por dificultad respiratoria. El modo del ventilador asisto controlado por volumen muestra los siguientes parámetros: $\mathrm{FiO}_{2}$ del $60 \%$, volumen corriente de $500 \mathrm{~mL}$, PEEP de $5 \mathrm{cmH}_{2} \mathrm{O}$, frecuencia respiratoria de 22 respiraciones/min y flujo inspiratorio de $80 \mathrm{~L} / \mathrm{min}$. La pa- ciente está muy sedada y no respira sobre las respiraciones programadas. Quince minutos después de la intubación, una gasometría revela $\mathrm{pH}$ de 7,22, $\mathrm{PaCO}_{2}$ de $60 \mathrm{mmHg}, \mathrm{PaO}_{2}$ de $85 \mathrm{mmHg}_{\text {y }} \mathrm{SaO}_{2}$ del 95\%. La presión pico es de $70 \mathrm{cmH}_{2} \mathrm{O}$, y la presión meseta, de $40 \mathrm{cmH}_{2} \mathrm{O}$. Su presión arterial es de $85 / 60 \mathrm{mmHg}$ y decreciendo. La frecuencia cardiaca se ha incrementado a 120 latidos/min (anterior de 95 latidos/min).

\section{La intervención más apropiada en este momento sería:}

a) Disminuir la frecuencia respiratoria a 12 respiraciones/min.

b) Aumentar la PEEP a $15 \mathrm{cmH}_{2} \mathrm{O}$.

c) Aumentar el volumen corriente a $600 \mathrm{~mL}$.

d) Disminuir la $\mathrm{FiO}_{2}$ al $30 \%$.

\section{8. ¿Cómo cuantificaría la cantidad de auto-PEEP presente en un paciente (que está sedado y paralizado)?:}

a) Medir la presión de la vía aérea durante una pausa de $1 \mathrm{~s}$ al final de la inspiración (y sustraer la PEEP establecida).

b) Medir la presión de la vía aérea durante una pausa de 1 al final de la espiración (y sustraer la PEEP establecida).

c) Restar la presión meseta de la presión pico.

d) Multiplicar el flujo por el volumen corriente.

En la mañana del segundo día de ventilación mecánica, mientras la paciente se encuentra muy sedada, la $\mathrm{SaO} 2$ disminuye repentinamente del $96 \%$ al $84 \%$ y la presión arterial cae de 118/76 a 90/55 mmHg. La presión pico de la vía aérea se ha incrementado de 40 a $75 \mathrm{~cm} \mathrm{H}_{2} \mathrm{O}$ y la presión meseta, de 28 a $50 \mathrm{cmH}_{2} \mathrm{O}$. La paciente permanece sedada. En el examen pulmonar hay presencia de sibilancias y existe buen movimiento de aire en el lado izquierdo, aunque se observa disminución en el lado derecho. Los sonidos cardiacos están distantes y regulares.

\section{9. ¿Cuál de las siguientes explicaciones es la más probable para los cambios en las presiones de la vía aérea?:}
a) Oclusión del tubo endotraqueal.
b) Aumento del broncoespasmo.
c) Asincronía paciente-ventilador.
d) Neumotórax a tensión. 